\title{
Distal renal tubular acidosis
}

INSERM

\section{Source}

INSERM. (1999). Orphanet: an online rare disease and orphan drug data base. Distal renal tubular acidosis. ORPHA:18

Distal renal tubular acidosis ( $\mathrm{dRTA}$ ) is a disorder of impaired net acid secretion by the distal tubule characterized by hyperchloremic metabolic acidosis. The classic form is often associated with hypokalemia whereas other forms of acquired dRTA may be associated with hypokalemia, hyperkalemia or normokalemia. 